\title{
DOUBLE-MODE STELLAR PULSATION
}

\author{
G. HOVACS \\ Department of Physics, liniversity of Florida. Gainestille \\ and \\ Fonkoly Observatory, Budapest, Hungary
}

\begin{abstract}
The current observational and theoretical status of the double-mode variables is reviewed. Focusing mostly on the RR Lyrae stars, we address the question of the observational evidence of modal stability. The problem of stationarity is a crucial issue in the modelling of these stars.

We mention past efforts in hydrodynamical and analytical modelling together with a detailed discussion of some very recent results. It is suggested that stochastic forcing due to turbulent convection may play a crucial role in exciting some marginally stable modes in the limiting pulsation. The latest hydrodynamical results first demonstrate that purely radiative models are able to show permanent double-mode behavior in the relevant period regime of RRd stars. The reason for the previous lack of double-mode behavior is attributed to the large dissipation, i.e. artificial viscosity, generally used in the codes to ensure numerical stability and to obtain amplitudes comparable to the observed ones.

We think that better models should include some physical dissipation, most probably turbulent convection, and a more accurate numerical treatment of the radiative hydrodynamics.
\end{abstract}

\section{Introduction}

Double-mode variables represent perhaps the 'cleanest' example of multimode stellar pulsation. Their light- (or velocity-) variations are described usually within the observational errors as a result of a Fourier-sum of two non-commensurable frequency components and their linear combinations. The two principal components are identified with some low-order, usually the fundamental and first overtone radial modes of pulsation. Simple linear pulsational models allowed already some 20 years ago to estimate the masses of the double-mode variables directly from their observed frequencies of pulsation. The development and perfection of this method and its application to multimode variables is called stellar seismology nowadays. The power of this method led to the initiative of revising the older stellar opacities and providing not only a better modelling of the atomic physics but also resolving the long-standing mass-discrepancy problems between the evolution and pulsation theories.

Based on the reasonable assumption of radial stellar pulsation, we can try to model finite amplitude double-mode pulsation. It is the basic nonlinear nature of double-mode pulsation and its modelling which are our main concerns in this review.

Numerical modelling of double-mode pulsation is important for three main reasons: (1) double-mode variables are hoped to be modelled by $1-D$ hydrodynamical codes, unlike the majority of the multimode pulsators which 
are non-radially pulsating stars; (ii) modelling means fine tuning the physics and the numerical methods we use in solving the physical problem, and perhaps also narrowing down further the model parameters; (iii) successful numerical modelling is the only way to reach an understanding of the underlying physics of double-mode stellar pulsation and to build to a simple model of it.

As it is well known, so far, the hydrodynamical models have failed to give permanent double-mode behavior in the observed ranges of periods. One of the main purposes of this paper is to summarize the results of some very recent tests which show that purely radiative RR Lyrae models do exhibit sustained double-mode pulsation in the right period range if the artificial viscosity is reduced enough.

The outline of this paper is the following. After a brief review of the current observational status of the double-mode variables in Section 2, we mention the effect of the new opacities on the period ratio masses in Section 3. Some possible phenomenological models with the implication of the dynamical effects of noise are discussed in Section 4. The past hydrodynamical simulations together with the very recent ones are reviewed in Section 5. Finally, in Section 6 we summarize our conclusions and highlight the important questions.

\section{Observations}

Since the primary purpose of this review is to deal with the theoretical aspects of double-mode pulsation, here we merely mention the main observational features which are important for the nonlinear modelling.

\subsection{Classical Cepheids}

The observational properties of the beat Cepheids have been reviewed recently by Balona (1985) and Szabados (1988). It is remarkable that about $30 \%$ of the observed galactic Cepheids are double-mode in the period range of 2-4 days, whereas no definite identification of this type of variables has yet been made in any extragalactic objects (see however Andreasen 1988). There is no preferred range in mode amplitude ratios, although most of the beat Cepheids have large fundamental than first overtone amplitudes. There is no convincing evidence for amplitude change on a long-time scale (Balona 1985). Even more, some stars show remarkably stable light variation on a 50-70 years time base (Jerzykiewicz 1988).

\subsection{Dwarf Cepheids}

The large-amplitude pulsators situated in the lower portion of the Cepheid instability strip close to the main sequence constitute an inhomogeneous group of stars, often called dwarf Cepheids. Some of them are very similar 
to the $\delta$ Scuti stars, but there are a few with distinctly Pop II characteristics. For a recent observational review of mostly the single-mode Pop II dwarf Cepheids we refer to Nemec and Mateo (1990), and for a more general review to Fitch (1980). The two principal components of the pulsation are generally assumed to be the radial fundamental and first overtone modes. The important question of amplitude stability has not yet been seriously addressed for these variables. There are, for example irregularities which are not explained yet (e.g. Fernley $\epsilon t$ al. 1987). It is possible, that some higher order overtones are somehow excited with low amplitudes which cause the seemingly irregular behavior. The exciting new analysis of AI Vel by Walraven, Walraven and Balona (1992) seems to support this view. They identify altogether four (or possible five) modes of pulsation. The newly discovered modes have very small $(\lesssim 10 \%)$ amplitudes compared to the principal ones. There are also some fluctuations in the amplitudes of these modes. The discovery of these modes is very significant, especially if some of them will be proved to be radial modes.

\subsection{RR LYRAE'STARS}

Until 1983, the field star $A Q$ Leo and the M3 variables V67 and V87 were the only known double-mode RR Lyrae (RRd) stars (Jerzykiewicz and Wenzel 1977; Goranskij 1981). The discovery of a large number of RRd stars in the globular cluster M15 by Cox, Hodson and Clancy (1983) initiated a number of subsequent investigations in other clusters and galaxies. According to the periods, the RRd stars form two distinct groups, following largely the overall metal abundance of the cluster. The low-metal (Oosterhoff II) RRd stars have $P_{0}=0.55 \pm 0.02$ day, $P_{1} / P_{0}=0.746 \pm 0.001$, whereas those in the high-metal (Oosterhoff I) clusters have $P_{0}=0.48 \pm 0.005$ day, $P_{1} / P_{0}=$ $0.7444 \pm 0.0004$. There are altogether $16 \mathrm{Oo}$ I and 24 Oo II RRd stars securely identified and some more suspected. A distinct property of all the RRd stars is that their first overtone amplitudes are a few times larger than those of the fundamental. The only exception is variable V68 in M3, where the two amplitudes are about the same (Nemec and Clement 1989). There have been no significant long-term amplitude changes reported in any RRd star (Kovács, Shlosman and Buchler 1986; Nemec and Clement 1989; Jurcsik and Barlai 1990). AQ Leo, the only RRd star observed photoelectrically, showed remarkable stability ( $\Delta A \leq 0.005 \mathrm{mag}$.) during three years of observation (Jerzykiewicz and Wenzel 1977).

One great mystery about the RRd stars is their occurrence and frequency. Many careful analyses have been made during the past several years based mostly on old photographic materials. The searches have largely been unsuccessful (see Clement and Walker 1991 and references therein). The complete absence of RRd stars in the cluster $w$ Cen which has 155 known RR Lyrae stars, or the only 2 RRd stars found among the $180 \mathrm{RR}$ Lyrae stars of M3, in- 
dicate that double-mode variability is not all common among the $R R$ Lyrae stars and may actually be a very delicate phenomenon, which depends on some, so far unknown fine details of stellar structure and evolution.

For a more detailed review of the observational aspects of the RRd stars we refer to Szeidl (1988).

\section{Period Ratio Masses}

The very powerful method of mass determination with the aid of the observed periods of double-mode variables (e.g. Petersen 1973) has triggered a lot of discussions and arguments on the discrepancies between the pulsation and evolution theories ( $c f$. Cox 1987). Among the many, sometimes exotic proposals to resolve this discrepancy, Simon's (1982) hypothesis on the possible underestimation of heavy element opacities has proved to be the most fruitful one. Due mainly to this idea, a substantial effort for updating the equations of state and opacities for stellar conditions has led to the first results in the last year (Iglesias and Rogers 1991; Rogers and Iglesias 1992). Now it seems that all major discrepancies between the evolution and pulsation theories have been eliminated. However, a stronger sensitivity to the heavy element abundance leaves us with fairly large uncertainties in some cases.

Moskalik, Buchler and Marom (1991) studied the beat and bump Cepheids with the new opacities of Iglesias and Rogers (see also Zalewski 1992). Moskalik et al. conclude that for beat Cepheids the period ratio masses are between $4-7 M_{\odot}$, in essential agreement with the standard evolution theories. For bump Cepheids, the derived masses are still somewhat low, but only if we take some moderate heavy element abundance $(Z=0.02)$ and a standard evolutionary mass - luminosity $(M-L)$ relation. We think that the errors both in $Z$ and in the evolutionary $M-L$ relations are high enough to render the remaining small discrepancy insignificant.

As for the RRd stars, the new opacities completely ruined our previous picture about these stars. Even for the low $Z$ Oo II variables the new opacities predict a mass of $\approx 0.77 M_{\odot}$, which is $\approx 0.1 M_{\odot}$ higher than the old value. Because of their higher $Z$, Oo I variables tend to have the same mass as the Oo II stars (Kovács, Buchler and Marom 1991; see also Cox 1991). In addition to the very strong $Z$ dependence of the derived mass, there is a substantial sensitivity even to the detailed mixture of the heavy elements. In a recent paper Kovács $\epsilon$ al. (1992) discuss this mixture dependence within the framework of the latest observational and theoretical results regarding the chemical compositions of Pop II stars (for a review, see Wheeler, Sneden and Truran 1989). They conclude that present inaccuracies in the observed chemical compositions and the lack of direct measurements on RRd stars, prevent us from estimating their masses on the basis of their periods more 
accurately than $\pm 0.1 M_{0}$. For this reason, period ratio masses are not very useful at the present moment for making a more thorough comparison with the evolution studies. More accurate direct chemical composition measurements are indispensable to make further progress here.

\section{Phenomenological Models}

The basic nonlinear behavior of a pulsating star is described by a set of ordinary differential equations which refer to the dynamical evolution of the amplitudes of the normal modes ( $c f$. Buchler 1985). However, except for second order adiabatic coupling, the nonlinear parameters entering in the amplitude equations are very difficult to compute $a b$ initio from the stellar models. Some information can be obtained through a comparison with the nonlinear hydrodynamical models, but in general the nonlinear coupling coefficients are regarded as 'almost' free parameters. Therefore, most of the studies on the amplitude equations can be considered as phenomenological. It is important however, that in some cases one can derive strict and general results without invoking the specific value of the coupling coefficients.

In the following we summarize the basic conclusions obtained from the amplitude equations relevant for the double-mode problem. First we review the cases when the system does not contain any stochastic forcing. The effect of the additive noise on the non-resonant pulsation is to be discussed in the second part of this section.

\subsection{DOUBLY-PERIODIC SOLUTIONS IN NOISELESS SYSTEMS}

\section{(1) Non-Resonant System}

Two modes coupled in a non-resonant way may settle down on a singleor double-mode state. The two possibilities are mutually exclusive in the sense that for a given set of parameters either one or two single-mode states or only one double-mode state can physically exist. More specifically, if we assume (supported by hydrodynamical results) that the nonlinear coupling coefficients are constants and negative across the instability strip, we can classify the solutions in two distinct groups, depending solely on the nonlinear coupling. Crossing the instability strip from the blue to the red, we have the following set of states.

Case (1): first overtone only - either first overtone or fundamental - fundamental only

Case (2): first overtone only - double-mode only - fundamental only

One of the major practical conclusion we can draw from these results is that non-resonant double-mode behavior is unique, there is no hysteresis which includes this state. For further discussion of the non-resonant mode coupling we refer to Dziembowski and Kovács (1984) and Buchler and Kovács (1986). 
(2) Resonant system

We consider only the lowest order of resonances. Depending on the number of modes involved in the resonance there are two types of interaction.

(2A) TwO-MODE: $2 \omega_{0} \approx \omega_{j}$

The importance of the $2: 1$ resonance in stellar pulsation was first noticed by Simon and Schmidt (1976). In the case of classical Cepheids this type of resonance between the fundamental and second overtone modes gives rise to the specific progression of Fourier parameters (i.e. Hertzsprung progression, see Buchler, Moskalik and Kovács 1990). The possibility that the same type of resonance may also affect the stability of the single-mode state, thereby leading to double-mode pulsation, was recognized by Dziembowski and Kovács (1984).

The basic mechanism is the amplitude decreasing effect of the resonance. For example, if there is a resonance between the fundamental and a high overtone, the amplitude of the fundamental limit cycle decreases and reaches a minimum at or near the center of resonance. As a result of it, the stability of this limit cycle decreases, and may allow other, linearly unstable modes (most importantly the first overtone) to grow in the limit cycle. The decrease of the stability of the resonant limit cycle is a general consequence of the resonance and can be seen both in the analytical considerations and in the numerical results (Kovács and Buchler 1988). If the fundamental limit cycle becomes unstable and the resonant first overtone limit cycle is also unstable, there is a three-mode (but because of phase lock, doubly-periodic) state which is the only stable state of the system. In the general case of nonadiabatic coupling the situation in principle could be more complicated, but numerical results support the simple picture we described.

(2B) ThreE-MODE: $\omega_{0}+\omega_{1} \approx \omega_{j}$

Simon (1979) suggested that this type of resonance may play a role in the double-mode pulsations of Cepheids and dwarf Cepheids. Subsequent hydrodynamical and analytical studies, however, did not support this hypothesis (Simon, Cox and Hodson 1980; Dziembowski and Kovács 1984). The following rigorous analytical results obtain.

Assuming that the fundamental and first overtone modes are linearly excited, while the resonant high overtone is damped and that there is resonant adiabatic coupling only, then, the three-mode resonance is never able to establish stable pulsation with constant amplitudes. When nonresonant coupling is also included, one of the single-mode states remains always stable while a stable three-mode state may occasionally exist simultaneously with the single-mode state for some very restricted range of parameters.

The general case (including non-resonant and non-adiabatic couplings) 
was discussed by Kovács and Kolláth (1988). Here we can observe the whole spectrum of nonlinear behavior of a dynamical system. Since there are no direct computations regarding the non-adiabatic effects in the resonant coupling and there are no hydrodynamical simulations which indicate the importance of three-mode resonance, we think that the role of this resonance remains hypothetical.

For completeness, we remark that the three-mode resonance may play an important role in the case of low-amplitude pulsators ( $\delta$ Scuti, Ap stars, white dwarfs). In $\delta$ Scuti stars, the low frequency modes correspond to higher overtone $g$-modes which interact with the low-order $p$-or $g$-modes. This parametric excitation was investigated by Dziembowshi and Krolikowska (1985), Dziembowski, Krolikowska and Kosovitchev (1988) and by Moskalik (1985).

\subsection{Noise Generated Multimode States}

There is no physical system which is free of random perturbations. This is particularly true for stars, where turbulent convection may exert a substantial effect not only on the static (i.e. average) structure, but on the pulsation too. There are two types of convective dynamical effects: (1) The part of the stochastic interaction which has non-vanishing ensemble average changes the dynamics in a deterministic way. The classical treatment of pulsation-convection interaction with the mixing length theory deals with this problem (e.g. Stellingwerf 1984); (2) The stochastic forcing which has zero ensemble average establishes a sustained perturbation of the system. One of the results of this is an excitation (usually at very small amplitudes) of all the normal modes of the system. This is what is claimed to happen in the case of the solar 5-min oscillations (Goldreich and Keeley 1977).

In this subsection we would like to elaborate further on the effects of stochastic mode excitation in the context of multimode nonlinear pulsation. Naturally, we are interested in systems which are close to some bifurcation, therefore, they are easy to influence even by a small amount of noise. We show, that for a sufficiently large number of stochastically excited modes the non-resonant interaction among these and the principal modes may lead to a genuine multimode state even if the noiseless system is unstable in that state. The fluctuation of the amplitudes of the principal modes can be arbitrary small depending on the number of modes included in the interaction.

Let us first consider the case when we omit all the modes except for the tow principal ones. Assuming additive noise in the original system and short noise correlation time, one can derive the following amplitude equations (Buchler, Goupil and Kovács 1992)

$$
\frac{d A_{0}}{d t}=\kappa_{0} A_{0}+Q_{00} A_{0}^{3}+Q_{01} A_{0} A_{1}^{2}+\frac{1}{2} \frac{S_{0}}{A_{0}}+\eta_{0}(t)
$$




$$
\frac{d A_{1}}{d t}=\kappa_{1} A_{1}+Q_{11} A_{1}^{3}+Q_{10} A_{1} A_{0}^{2}+\frac{1}{2} \frac{S_{1}}{A_{1}}+\eta_{1}(t) .
$$

Here $\eta_{0}(t), \eta_{1}(t)$ are the fluctuating non-parametric noise components which are easily related to the ones appearing in the original system. $S_{0}, S_{1}$ are the spectral densities of $\eta_{0}(t), \eta_{1}(t)$ at frequency zero. Some useful information can be obtained regarding the average values and the stability of the amplitudes by studying the system $(1)-(2)$ with $\eta_{0}(t)=\eta_{1}(t)=0$, but keeping the intensity terms. It follows immediately that we only have doublemode solutions because of the $S_{i} / A_{i}$ terms. These are, however, no genluine double-mode solutions, because as one can show by an analysis of the amplitude fluctuations, one of the two modes remains of precursor-type, i.e. its average amplitude and the fluctuation around it will be of the same size. For high enough noise the two double-mode solutions (associated with the noiseless single-mode states) can merge together and form a unique noisy double-mode state with one single maximum in the probability distribution function (see Buchler 1992).

To illustrate the behavior of the stochastic double-mode solution, we integrate Eqs. (1)-(2) for a parameter set which corresponds to a 'first overtone only' case in the noiseless system. The empirical probability distribution functions are shown in Fig. 1. Because of the large dispersion, it is clearly seen that the low-amplitude mode is a noise generated one. The smaller the absolute value of the switching rate of this mode is, the larger is the excited amplitude at a constant noise level. It is important to remark that though the amplitude has a large scatter, the variation of the signal is smooth on a time scale of some fraction of the dynamical evolution (i.e. $1 / \bar{\kappa}$, where $\bar{\kappa}$ is the switching rate).

Regarding the observed double-mode variables, the model discussed above is not acceptable, because of the large amplitude fluctuations of the precursor mode. It is clear, however, that the mechanism could be quite efficient to excite some other modes which are just marginally damped in the limit cycle or in the double-mode state. We conjecture that the observed irregularities in $\delta$ Scuti stars, dwarf Cepheids and maybe in other stars too, could quite possibly be attributed to noise excited higher order modes. We emphasize the fact that the amplitudes of these modes could be much larger than the ones maybe obtained by a solar analogy (Christensen-Dalsgaard and Frandsen 1983). The reason for this is that we deal with linearly excited modes which could be only very mildly damped in the limit cycle. The discovery of a few very low-amplitude modes in AI Vel (Walraven $\epsilon t$ al. 1992) is very important and deserves further attention from the theory of the stochastic mode excitation.

Let us now examine the case of multimode non-resonant interaction in the presence of noise. Our aim is to find situations in which the system bifurcates to a truly double-mode state in which the principal modes have very little 


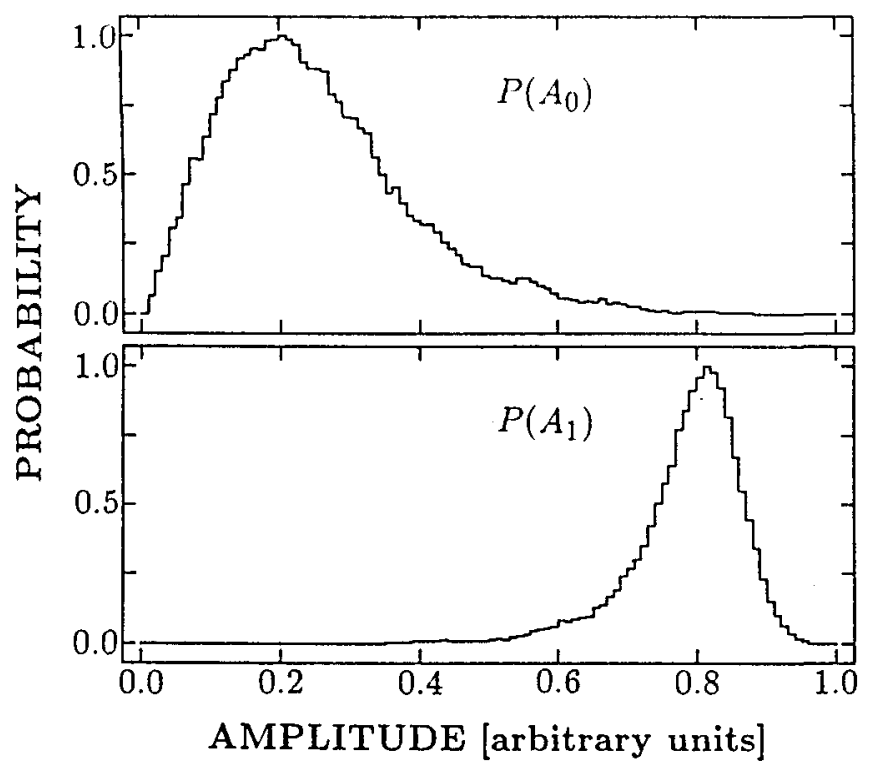

Fig. 1. Empirical probability distribution functions of the amplitudes after integrating Eqs. (1)-(2). The parameters are: $\kappa_{0}=0.01, \kappa_{1}=0.06, Q_{00}=-10, Q_{01}=-15$, $Q_{10}=Q_{11}=-50, S_{0}=S_{1}=10^{-6}$.

scatter. The details of this non-trivial problem are given by Buchler and Kovács (1992). Here we just mention the highlights of the solution.

Without jeopardizing generality, we assume that there are three modes only, that the first two are linearly excited and that the parameters correspond to a 'first overtone only' situation. Also, to obtain some analytically tractable expression, the noise is omitted in the first two modes $\left(S_{0}=S_{1}=\right.$ 0 ). The switching rates in the respective fundamental and first overtone limit cycles are

$$
\begin{aligned}
& \bar{\kappa}_{1}=\kappa_{1}+Q_{10} A_{0}^{2}+Q_{12} A_{20}^{2}, \\
& \bar{\kappa}_{0}=\kappa_{0}+Q_{01} A_{1}^{2}+Q_{02} A_{21}^{2},
\end{aligned}
$$

where $A_{2 j}$ is the amplitude of the noise excited third mode in the presence of the limit cycle $j$. The equilibrium solutions are given by

$$
\begin{aligned}
& \kappa_{j}+Q_{j j} A_{j}^{2}+Q_{j 2} A_{2 j}^{2}=0, \\
& \kappa_{2}+Q_{2 j} A_{j}^{2}+Q_{22} A_{2 j}^{2}+\frac{1}{2} \frac{S_{2}}{A_{2 j}^{2}}=0, \quad j=0,1 .
\end{aligned}
$$

Because $Q_{i j}<0$ for all $(i, j)$, it follows from Eq. (5) that the amplitudes of the principal modes decrease in the presence of this type of noise. Then, 
according to Eq. (3) and (4), the limit cycles may become less stable if the stabilizing effect of the $Q_{j 2} A_{2 j}^{2}$ terms does not overwhelm the opposite trend caused by the decrease of the limit cycle amplitudes. It is an important and interesting fact that one can readily find some parameters for which one of the switching rates decreases, while the other one increases (it is easy to show that the two switching rates cannot increase simultaneously). It means that this type of interaction cannot cause double-mode solution if the noiseless system is in the 'ether-or region' $\left(\bar{\kappa}_{0}<0, \bar{\kappa}_{1}<0\right)$. It is also clear that the coupling with the principal modes in Eq. (6) is very important, since otherwise only the effective linear growth rates are decreased, which does not alter the stability of the system.

Once a proper set of parameters is found with $\bar{\kappa}_{0}>0, \bar{\kappa}_{1}>0$, we can tune the noise and let the system bifurcate (in this specific case for example) from the first overtone (low noise, $A_{0}=0, A_{1} \neq 0, A_{j 1} \neq 0$ ) through doublemode (medium noise, $A_{0} \neq 0, A_{1} \neq 0, A_{j} \neq 0$ ) to fundamental (high noise, $\left.A_{0} \neq 0, A_{1}=0, A_{j 0} \neq 0\right)$.

In the case of many high-order modes we can redistribute the total highorder mode energy necessary to destabilize the quasi single-mode states among many modes. Then, since the individual mode energies become lower and their fluctuations are almost independent one can expect a lower dispersion for the principal modes. Some more detailed considerations do indeed show that this is the case. The r.m.s. scatter of the amplitudes of the principal modes changes as $1 / \sqrt{N}$, where $N$ is the total number of the high overtone modes.

To illustrate the existence of the true double-mode solution and the decrease of the amplitude dispersion as the number of the modes increase, we perform a similar numerical simulation as we have already done in the twomode case. The results are shown in Fig. 2. The noiseless system corresponds to a 'first overtone only' case and the nonlinear parameters to an 'either-or' case (i.e. no double-mode solution is possible for the noiseless system).

The difference between this and the precursor-type double-mode system is clearly shown (compare with Fig. 1). The simulations with a large number of modes exhibit the decrease of the amplitude dispersion in a quantitative agreement with the theoretical $1 / \sqrt{N}$ dependence.

An application of the above idea to hydrodynamical models is fairly straightforward. The coupling coefficients can be extracted from the limit cycle analysis and the result can be used directly to evaluate the analytical stability conditions. A large number of tests made with standard RR Lyrae models indicate that the required conditions up to the 6 -th order radial modes are not satisfied (at least not with 'standard' artificial viscosity). We think that the mechanism of noise generated 'smooth' multimode states should be further studied by exploring the effects of resonances. In any case as a partially ad hoc idea we cannot exclude that the nonradial modes, which 


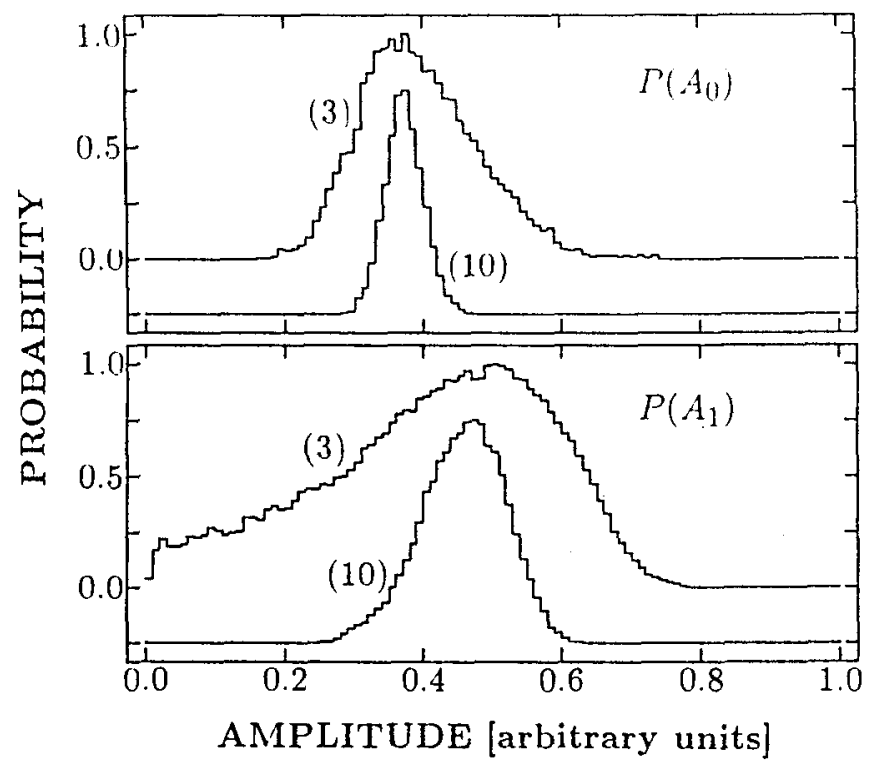

Fig. 2. Empirical probability distribution functions of the amplitudes of the principal modes in the case of multimode non-resonant interaction. The total number of modes are shown at the curves. The parameters are: $\kappa_{0}=0.01, \kappa_{1}=0.06, Q_{00}=-10, Q_{01}=-15$, $Q_{10}=Q_{11}=-50, \kappa_{j}=-0.12, Q_{0 j}=Q_{j 1}=-50, Q_{1 j}=Q_{j 0}=-500$, for $j \geq 2$ and all other $Q_{i j}$ are zero. The intensities are: $S_{0}=S_{1}=10^{-4} S_{j}, S_{j}=2 \times 10^{-5}$ and $2.5 \times 10^{-6}$ for the 3 and 10 mode case, respectively.

are most probably affected by turbulent convection, have proper couplings with the relevant radial modes. Then, a permanent double-mode state may exist, similarly as we have just described.

\section{Hydrodynamical Modelling}

Quite a few attempts have been made for numerical modelling of doublemode stars. After the early works (Stobie 1969; King et al. 1973), Stellingwerf (1975) was the first who, with the aid of the relaxation technique, performed the first systematic study in the limit cycle stability of the RR Lyrae stars. He found some cold models which study in the limit cycle stability of the RR Lyrae stars. He found some cold models which exhibited double-mode behavior. It is not only that those models have not much common with the observed double-mode stars, but also, subsequent simulations by Cox, Hodson and Davey (1976) could not confirm their double-mode nature. This is not very surprising in retrospect, since nonlinear behavior is very sensitive to some details of the model, which were certainly not identical in the two works.

Simon, Cox and Hodson (1980) tried to test the effect of three-mode 
resonance in supporting beat Cepheid pulsation. It turned out (in agreement with the subsequent analytical studies, as we have already mentioned) that this resonance is not likely to be important in double-mode pulsation.

The discovery of a large number of RRd stars further stimulated the search for double-mode pulsation models. Cox (1982) and Hodson and Cox (1982) made a moderate survey of RR Lyrae pulsation. They were unable to construct any RRd models. Re-investigating the problem, Kovács and Buchler (1988) performed a more extensive survey. They indeed found, in agreement with the analytical prediction of Dziembowski and Kovács (1984), that models which are in the proximity of the $2: 1$ resonance between the fundamental and the third overtone, exhibit less stable fundamental limit cycles compared to the ones outside of this resonance. This is a general property and is independent of the numerical details of the model. For some specific parameters, the resonance is able to destabilize the limit cycle and. if the first overtone (non-resonant) limit cycle is also unstable, lead to a doublemode model. The destabilizing effect of the 2:1 resonance has manifested itself in other types of models too, in particular, in Cepheid models (Buchler, Moskalik and Kovács 1990). Except for the fact that these models are the first, well established simulations showing permanent double-mode behavior, they are not relevant from practical point of view, because their periods are very different from the observed ones.

Convection, of course, may play an important role in the limit cycle properties of the models. Unfortunately, there has been no systematic and accurate work done in this area, except maybe for that of Ostlie (1990). He found that some of this RR Lyrae models indicate simultaneously unstable limit cycles. This observation was, however, not conclusive because the analysis was made on the perturbed, not perfectly settled limit cycles, and there was no subsequent direct time-integration performed to prove that the model really settles down on a double-mode state. Until the numerical problems are solved and more systematic studies are made on convective models, the role of convection in double-mode pulsation remains open.

Turning back to the purely radiative models, we would like to examine the effect of the artificial viscosity on the stability of the limit cycles. According to Stellingwerf (1975), the viscous pressure is given by

$$
\begin{aligned}
p_{v}(i) & =C_{Q} P_{g}(i)[(u(i-1)-u(i)) / c(i)-\alpha]^{2}, \\
& \quad \text { if } \quad(u(i-1)-u(i)) / c(i)>\alpha>0 \\
& =0, \quad \text { otherwise. }
\end{aligned}
$$

Here $c(i)=\sqrt{p_{g}(i) v(i)} ; p_{g}(i), v(i), u(i)$ are the gas pressure, the specific volume and the velocity of the $i$-th mass shell. It is well known that the artificial viscosity has an important role in determining the limit cycle properties. Since there is not much of a restriction on the viscosity parameters 
$\left(C_{Q}, a\right)$, one cannot justify to fix them to any particular value. One possible methodology is that we are interested in the behavior of the pure radiative model and therefore, we should avoid 'unphysical' dissipation as much as possible. The problems which arise here are that: (1) amplitudes might become too high, (2) numerical instabilities might get amplified. To handle these problems, previous nonlinear works employed a numerical viscosity, which seemed to be a fair trade between numerical stability, size of amplitudes and avoiding very large dissipation. Here we would like to relax the restriction regarding the size of the amplitudes, while maintaining numerical stability. The main goal of this test is to indicate some type of behavior of the models which could be observed in the simulations with future less dissipative and more accurate codes. A very detailed discussion of our tests is given in Kovács and Buchler (1992). Additional tests regarding the artificial viscosity are presented in Kovács (1990).

To illustrate the dramatic effect of decreasing the viscous dissipation we compute a sequence of RR Lyrae models with two different artificial viscosity parameters. The sequence has the following parameters: $M=0.75 M_{\odot}$, $L=40 L \odot, X=0.7, Z=0.0001, T_{\text {eff }}=6000-6400$. We use the opacities published by Rogers and Iglesias (1992). Unless stated otherwise, all of our models contain 60 mass shells. The results are shown in Fig. 3.

The following important observations can be made: (1) The switching rate from the fundamental limit cycle toward the first overtone $\left(\bar{\eta}_{1}\right)$ is strongly affected by the artificial viscosity. For the less dissipative $\left(C_{Q}, \alpha\right)=(4,0.07)$ models $\bar{\eta}_{1}$ has a non-monotonic behavior and a very large positive value in an extended region. (2) The stability of the first overtone limit cycle is not much affected; the switching rate toward the fundamental $\left(\vec{\eta}_{0}\right)$ does not change appreciably. (3) There is a region in which both switching rates are simultaneously positive. (4) The amplitudes of both limit cycles increase considerably for the less dissipative models.

We see that a completely different behavior is obtained for lower artificial dissipation. The 'standard' viscosity parameters $\left(C_{Q}, \alpha\right)=(4,0.01)$ would predict a transition from an 'either - or' region $\left(T_{\text {eff }}>6250\right)$ to a 'fundamental only' one $\left(T_{\text {eff }}<6250\right)$, whereas for lower viscosity we get a 'double-mode only' regime ( $\left.T_{\text {eff }}=6250-6080\right)$ sandwiched between the two different singemode regimes. This behavior survives for many other $\left(C_{Q}, a\right)$ combinations, when the resulting viscous dissipation is small enough. It is also comforting to know that the periods and period ratios in the double-mode region overlap with those of the Oo II RRd variables, namely: $P_{0}=0.51-0.56$ day, $P_{1} / P_{0}=0.746-0.747$ for our double-mode models.

The puzzling question regarding the behavior of $\bar{\eta}_{1}$ is that it has a nonmonotonic variation and that it has a much larger value than that of the corresponding linear growth rate. None of these properties are easily explained within the framework of non-resonant pulsation (see e.g. Buchler, 


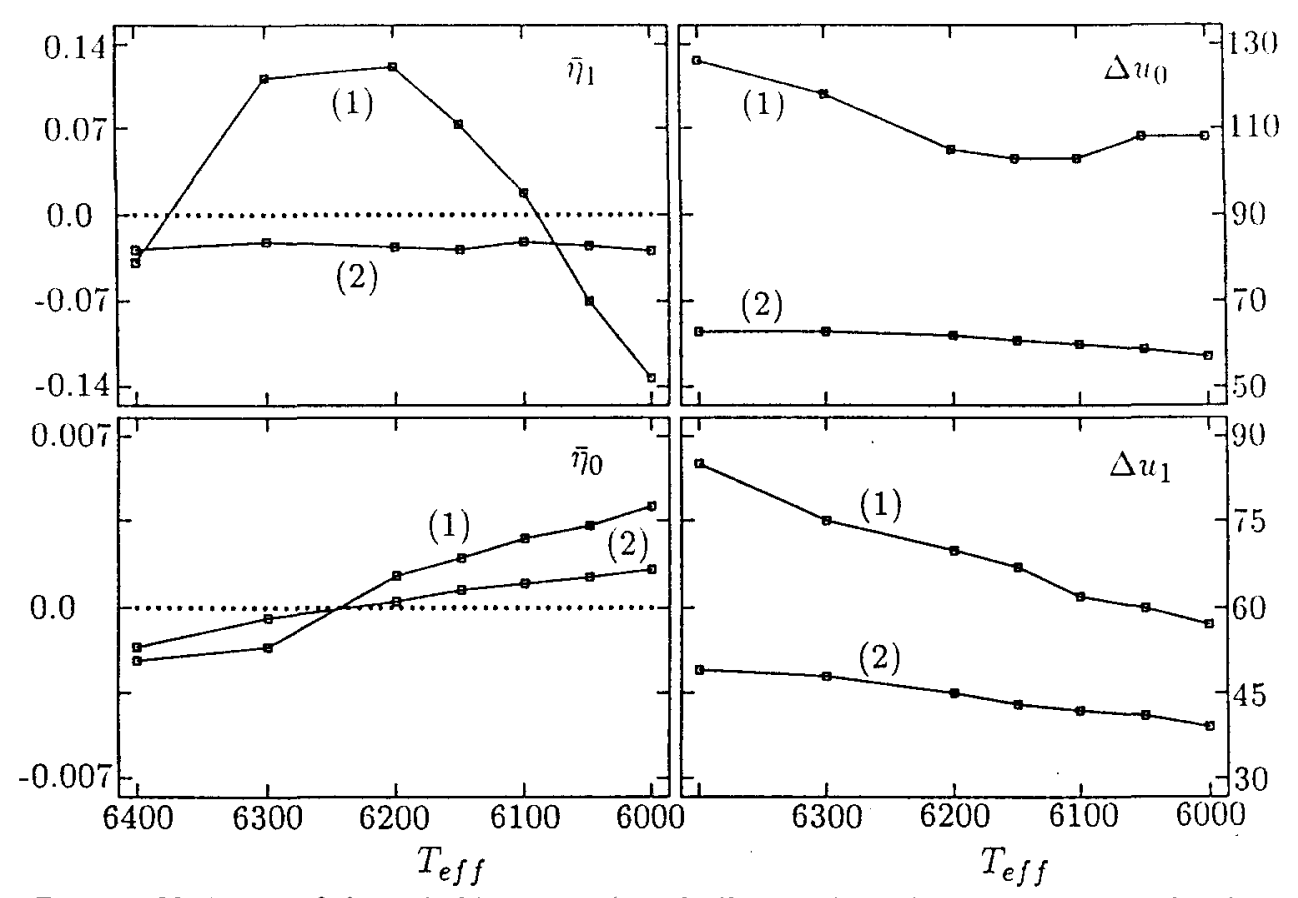

Fig. 3. Variation of the switching rates (e.g. Stellingwerf 1975) and velocity amplitudes (in $\left[\mathrm{kms}^{-1}\right]$ ) along a model sequence with $M=0.75 M_{\odot}, L=40 L_{\odot}, Z=0.0001$. Numbers at the lines denote the various values of the artificial viscosity parameters: (1): $C_{Q}=4$, $\alpha=0.07 ;(2) C_{Q}=4, \alpha=0.01$.

Moskalik and Kovács 1991). Therefore, we look for some type of resonance which leaves the system intact, except for the stability of the fundamental limit cycle. There are two different resonances in the proximity of the hump of $\bar{\eta}_{1}, v i z .3 \omega_{0} \approx \omega_{1}+\omega_{2}$ and $2 \omega_{1} \approx \omega_{0}+\omega_{2}$, where $\omega_{0}, \omega_{1}$ and $\omega_{2}$ are the fundamental, first and second overtone frequencies, respectively. The resonance centers are at $T_{\text {eff }} \approx 6300$ and $T_{\text {eff }} \approx 6100$ for the first and second resonance, respectively. Other more 'standard' resonances (two- and three-modes) are much 'weaker' in a numerological sense.

Which of these two resonances might cause the variation of $\bar{\eta}_{1}$ ? It is easy to see that the amplitude equation for $A_{1}$ is modified by the second resonance through a term which is proportional to $A_{0} A_{1} A_{2}$, consequently, the stability of the fundamental limit cycle will not be affected by this resonance, since $A_{0} A_{1} A_{2}$ is a higher order term, and therefore is negligible in the computation of $\eta \bar{\eta}_{1}$.

On the other hand, the first resonance $3 \omega_{0} \approx \omega_{1}+\omega_{2}$ contributes to the amplitude equations for $A_{1}$ and $A_{2}$ by terms proportional to $A_{0}^{3} A_{2}$ and $A_{0}^{3} A_{1}$, respectively. Therefore, this resonance will affect the stability of the fundamental limit cycle. As we see. the perturbations with the first and 


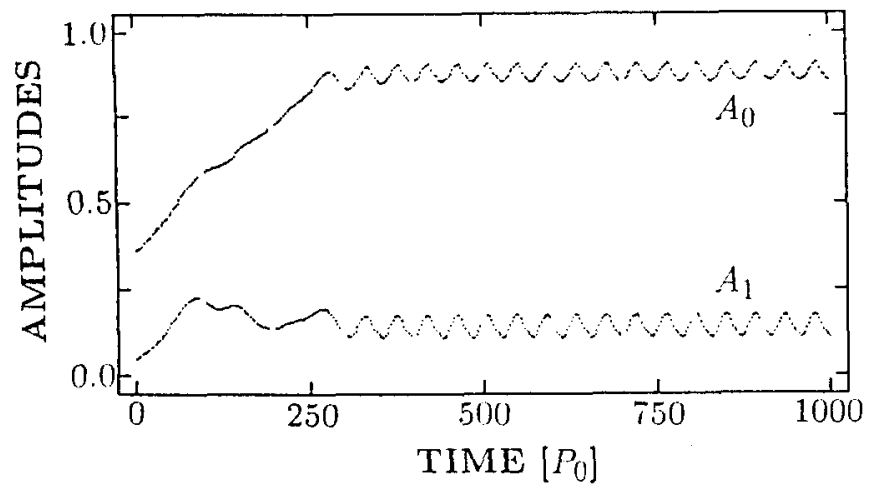

Fig. 4. Amplitude evolution of the radius variation of the $P_{0}=0.529$ day, $M=0.75 M_{\odot}$, $L=40 L_{\odot}, T_{\text {eff }}=6200 \mathrm{~K}, Z=0.0001$ model with $C_{Q}=4, \alpha=0.07$. The amplitudes are given in arbitrary units.

second overtones are combined, a property which is also clearly observable in the Floquet analysis of the numerical models. The limit cycle amplitudes are not affected by this resonance in agreement with the data plotted in Fig. 3 .

We now turn to the direct time integrations of the models. Each model is started from the static solution perturbed with a velocity distribution containing a mixture of first overtone (10\%) and fundamental (90\%) eigenmodes. The modal content of the radius or light variation is monitored by time-dependent Fourier analysis. One typical amplitude evolution is shown in Fig. 4. The double-mode state is independent of the initial conditions as is indicated by some supplementary tests.

It is interesting to note the periodic oscillations of the amplitudes, which is not an artifact of data analysis, but an inherent property of most of the models. This behavior is again an indication of the resonance discussed above.

Comparing with the observations one notes that the models have inverted amplitude ratios. The question is how we can reverse it? At the moment our guess is that some fine tuning of the dissipation together with a better spatial resolution might cause the desired effect.

In Fig. 5 we show the integrations of a model with $M=0.75 M_{\odot}$, $L=35 L_{\odot}, T_{\text {eff }}=5900 \mathrm{~K}, Z=0.0001$. In both cases the single-mode states are unstable, and therefore, the final states are surely double-mode states. We see that in the more dissipative case (lower panel), despite of the long integration, the system has still not reached equilibrium, but that the amplitude ratio is reversed. Except for the imperfectly matched periods $\left(P_{0}=0.56\right.$ day, $\left.P_{1} / P_{0}=0.744\right)$ the more dissipative model reproduces all directly observable main features of all Oo II RRd star. (Although our values for $L$ and 


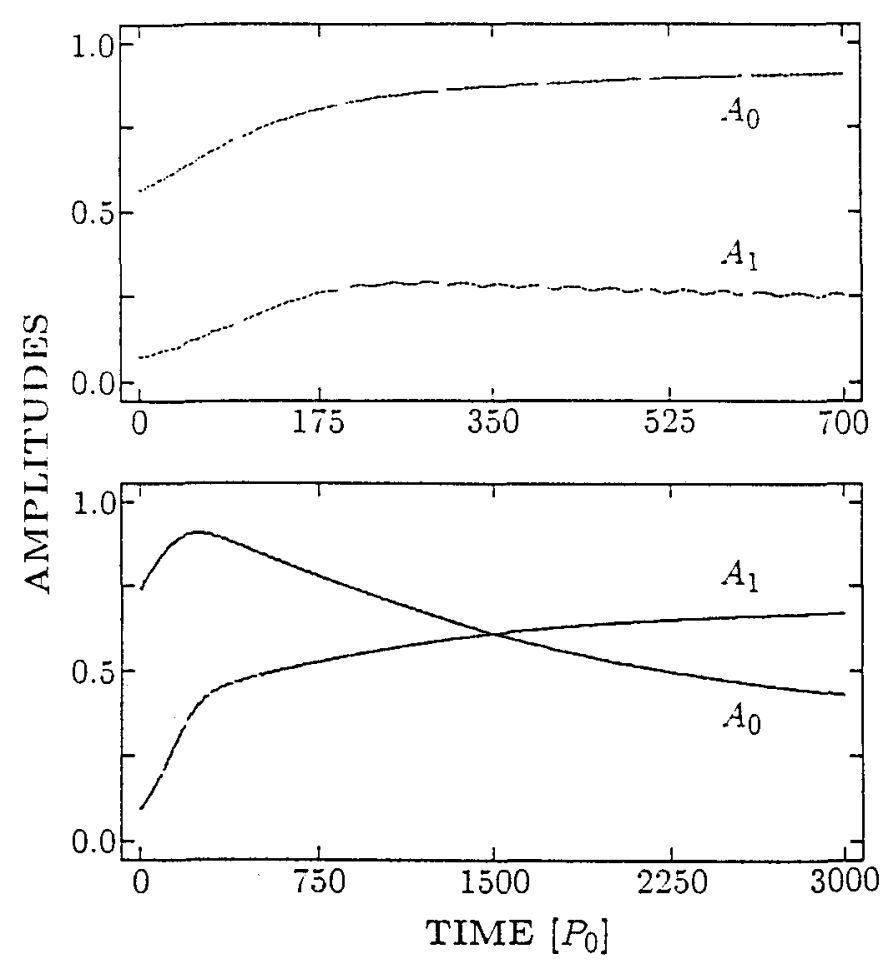

Fig. 5. Amplitude evolution of the radius variation of the $P_{0}=0.561 \mathrm{day}, M=0.75 . \mathrm{M}$, $L=35 L_{\odot}, T_{\text {eff }}=5900 \mathrm{~K}, Z=0.0001$ model with $C_{Q}=1, \alpha=0.004$ (upper panel) and $C_{Q}=1, \alpha=0.0$ (lower panel). The amplitudes are given in arbitrary units.

$T_{\text {eff }}$ are certainly lower for this model than the 'standard' one, we consider this discrepancy of a secondary importance. These non-direct observables contain large uncertainties, therefore, they enter with lower weights in a comparison with the observations.)

To illustrate the persistence of the double-mode behavior in the case of higher spatial resolution, we integrate a 90 zone model. The parameters are: $M=0.75 M_{\odot}, L=40 L_{\odot}, T_{\text {eff }}=6200 \mathrm{~K}, Z=0.0 ;$ i.e. except for $Z$, this is the same model as shown in Fig. 4 . The artificial viscosity parameters are also different, namely $\left(C_{Q}, \alpha\right)=(2,0.006)$ in contrast to $(4,0.07)$ used in the simulation shown in Fig. 4. We note that in the present form of the artificial viscosity, its effect on the behavior of the model depends on the number of zones. The same viscosity parameters for a finer zoned model cause larger amplitudes and possibly more violent behavior than for the coarse zoned model. The amplitude evolution shown in Fig. 6 proves that the double-mode behavior is maintained for higher zoned models too, without destroying the numerical stability and even improving the amplitude ratio.

We conclude this section by emphasizing that the latest simulations with 


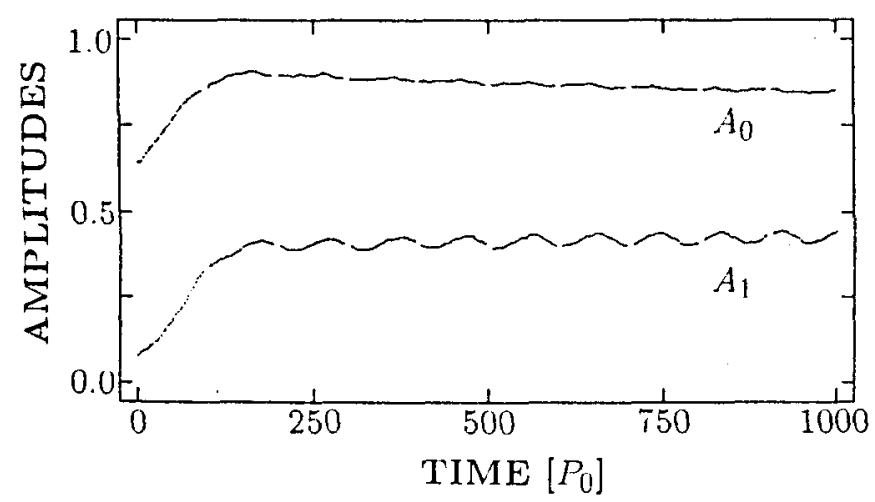

Fig. 6. Amplitude evolution of the light variation of a 90 zone $P_{0}=0.529$ day, $M=0.75 M_{\odot}, L=40 L_{\odot}, T_{\text {eff }}=6200 \mathrm{~K}, Z=0.0$ model with $C_{Q}=2, \alpha=0.006$. The amplitudes are given in arbitrary units.

a less dissipative purely radiative code strongly indicate that permanent double-mode behavior is possible at least for RRd models. These simulations are by no means devoted to modelling the detailed nature of doublemode variables, but rather are meant to stimulate further investigations with other, more accurate and less dissipative codes.

\section{Conclusions (and Questions)}

Understanding double-mode variables is one of the main objectives of the theory of nonlinear stellar pulsations. The simple and indisputable observational constraints (periods and amplitudes) put clear criteria on the acceptance of different models. The reasonable assumption of purely radial pulsation gives us the hope to model this simplest type of multimode pulsation with 1-D hydrodynamical codes. The achievement of this goal is still in the more distant future for the vast majority of multimode pulsators which are oscillating in non-radial modes.

Though the observations are fairly extensive in some cases, there are many unanswered questions, that could fairly simply be answered by employing more accurate (but still standard) observational techniques. More specifically, some of the questions to be addressed are as follows.

(1) Stability of the light variation. Especially for globular cluster RRd stars the accuracy of the photographic observations do not allow us to make any strict statement about this problem. If there were sizable fluctuations in the amplitudes, the theoretical interpretation of the doublemode variables could be quite different from the one we have for a pulsation of constant amplitude.

(2) Are there any other modes besides the two principal ones excited in 
double-mode variables? The beautiful new analysis of AI Vel by Walraven $\epsilon t$ al. (1992) shows that other modes at much lower amplitudes may well be excited. We think it is needless to emphasize the theoretical importance of the discovery of these low-amplitude modes. If some of them are radial modes then simple linear theory puts very strict restrictions on the parameters of the model. Excitation of these modes should tell us also something about the mechanism of double- (now multi-) mode pulsation.

(3) Statistics of the double-mode stars. What is the relation between cluster properties and the presence (or absence) of RRd stars? Why do we have only one RRd star in our galaxy? How many dwarf and beat Cepheids are in extragalactic objects?

(4) Is there any relation between some basic physical properties (like chemical composition, rotational velocity) and double-mode pulsation?

From the theoretical side, there is certainly a lot more to do in finding the cause of double-mode behavior and construct physically sound models. There are, however, two encouraging developments which may lead to some progress in the very near future.

(a) Turbulent convection may drive some modes which are marginally stable in some limiting state (either single- or double-mode). This leads to the possibility of observing a few additional modes (including radial ones) besides the large-amplitude principal modes. The dwarf Cepheid AI Vel could be just the first example for this type of mode excitation.

(b) Decreased artificial viscosity dissipation leads to double-mode pulsations in current purely radiative $R R$ Lyrae models. The periods of the nonlinear models now fit the observed values with reasonable stellar parameters, but the amplitude ratio depends sensitively on the specific choice of the artificial viscosity parameters.

It is clear, there is now an even more urgent need to develop less dissipative, more accurate nonlinear codes. A progress has recently been made in this field (Dorfi and Feuchtinger 1991; Cox, Deupree and Gehmeyr 1991; Gehmeyr 1991) and there are certainly more results to be expected in the near future (Buchler and Marom 1992). In addition, because of the excessive amplitudes, there is not doubt that the unphysical artificial viscosity should be replaced by (or supplemented with) some physical mechanism, such as turbulent viscous dissipation. Though the correct treatment of turbulent convection in $1-D$ will obviously remain a problem for many years, some approximate parametrized model might be enough to cure the problem of the amplitudes. 


\section{Acknowledgements}

The author is grateful to the LOC of this conference, and especially to Dr. Mine Takeuti for the hospitality and for the financial support. The travel grant from the Institute for Fundamental Theory in the Physics Department of the University of Florida is also very much acknowledged. Fruitful discussions with Robert Buchler and Ariel Marom were very useful during the preparation of this paper. This work was supported by NSF (AST 8914425 ) and by an RCI grant through IBM and the NER Data Center at the University of Florida.

\section{References}

Andreasen, G. K.: 1988, Astronomy and Astrophysics 191, 71.

Balona, L. A.: 1985, in Cepheids: Theory and Observations, Proc. IAU Coll. No 82, ed. B. F. Madore (Cambridge University Press), p. 17.

Buchler, J. R.: 1985, in Chaos in Astrophysics, NATO ASI Ser. C, Vol. 161, eds. J. R. Buchler, J. M. Perdang and E. A. Spiegel (Dordrecht: Reidel), p. 137.

Buchler, J. R.: 1992, these proceedings.

Buchler, J. R. and Kovács, G.: 1986, Astrophysical Journal 308, 661.

Buchler, J. R. Kovács, G.: 1992, Physica D, submitted.

Buchler, J. R., Goupil, M.-J. and Kovács, G.: 1992, Astronomy and Astrophysics, submitted.

Buchler, J. R. and Marom, A.: 1992, in preparation.

Buchler, J. R., Moskalik, P., and Kovács, G.: 1990, Astrophysical Journal 351, 617.

Buchler, J. R., Moskalik, P., and Kovács, G.: 1991, Astrophysical Journal 380, 185.

Christensen-Dalsgaard, J. and Frandsen, S.: 1983, Solar Phys., 82, 469.

Clement, C. M. and Walker, I. R.: 1991, Astronomical Journal 101, 1352.

Cox, A. N.: 1982, in Pulsations in Classical and Cataclysmic Variable Stars, eds. J. P. Cox and C. J. Hansen (Boulder: JILA), p. 157.

Cox, A. N.: 1987, in The Second Conference on Faint Blue Stars, eds. A. G. D. Philip, D. S. Hayes and J. W. L. Liebert (L. Davis Press, Inc., Schenectady, New York), p. 161.

Cox, A. N.: 1991, Astrophysical Journal, Letters to the Editor 381, L71.

Cox, A. N., Deupree, R. G. and Gehmeyr, M.: 1991, in Experimental Mathematics: Computational Issues in Nonlinear Science, Proc. of the CNLS 11 th Annual Conference, held in Los Alamos, May 20-24, 1991 (preprint).

Cox, A.N., Hodson, S. W. and Clancy, S. P.: 1983, Astrophysical Journal 266, 94.

Cox, A. N. , Hodson, S. W. and Davey, W. R.: 1976, in Solar and Stellar Pulsation, eds. A. N. Cox and R. G. Deupree (Los Alamos: LA-6544-C), p. 188.

Dorfi, E. A. and Feuchtinger, M. U.: 1991, Astronomy and Astrophysics 249, 417.

Dziembowski, W. and Kovács, G.: 1984, Monthly Notices of the RAS 206, 497.

Dziembowski, W. and Krolikowska, M.: 1985, Acta Astr. 35, 5.

Dziembowski, W., Krolikowska, M. and Kosovitchev, A.: 1988, Acta Astr. 38, 61.

Fernley, J. A., Jameson, R. F., Sherrington, M. R. and Skillen, I.: 1987, Monthly Notices of the RAS 225, 451 .

Fitch, W.: 1980, Lecture Notes in Physics 125, p. 7.

Gehmeyr, M.: 1991, On Non-Lagrangian Computations of Convective RR Lyrae Stars, Ph. D. Thesis, University of New Mexico.

Goldreich, P. and Keeley, D. A.: 1977, Astrophysical Journal 212, 243.

Goranskij, V. P.: 1981, Inf. Bull. Var. Stars, No. 2007.

Hodson, S. W. and Cox, A. N.: 1982, in Pulsations in Classical and Cataclysmic Variable Stars, eds. J. P. Cox and C. J. Hansen (Boulder: JILA), p. 201. 
Iglesias, C. A. and Rogers, F. J.: 1991, Astrophysical Journal, Letters to the Editor 371. L73.

Jerzykiewicz, M.: 1988, in Multimode Stellar Pulsations, eds. g. Kovács. L. Szabados and B. Szeidl, Konkoly Observatory (Kultura: Budapest), p. 19.

Jerzykiewicz, M. and Wenzel, W.: 1977, Acta Astr. 27. 35.

Jurcsik, J. and Barlai, K.: 1990, in Confrontation Between Stellar Pulsation and Evolution, Astron. Society of the Pacific. Conf. Ser., vol. 11, eds. C. Cacciari and G. Clementini, p. 112.

King, D. S., Cox, J. P., Eilers, D. D. and Davey, W. R.: 1973, Astrophysical Journal 182, 859.

Kovács, G.: 1990, in The Numerical Modelling of Nonlinear Stellar Pulsations; Problems and Prospects, ed. J. R. Buchler (Kluwer, Dordrecht), p. 73.

Kovács, G. and Buchler, J. R.: 1988, Astrophysical Journal 324, 1026.

Kovács, G. and Buchler, J. R.: 1992, Astrophysical Journal, submitted.

Kovács, G., Shlosman, I., and Buchler, J. R.: 1986, Astrophysical Journal, 307, 593.

Kovács, G., Buchler, J. R., and Marom, A.: 1991, Astronomy and Astrophysics (Letters) 252, L27.

Kovács, G., Buchler, J. R., Marom, A., Iglesias, C. A. and Rogers, F. J.: 1992, Astronomy and Astrophysics (Letters), submitted.

Kovács, G. and Kolláth, Z.: 1988, in Multimode Stellar Pulsations, eds. G. Kovács, L. Szabados and B. Szeidl, Konkoly Observatory (Kultura: Budapest), p. 33.

Moskalik, P.: 1985, Acta Astr. 35, 229.

Moskalik, P., Buchler, J. R. and Marom, A.: 1991, Astrophysical Journal 385, 685.

Nemec, J. M. and Clement, C. M.: 1989, Astronomical Journal 98, 960.

Nemec, J. M. and Mateo, M.: 1990, in Confrontation Between Stellar Pulsation and Evolution, Astron. Society of the Pacific. Conf. Ser., Vol. 11, eds.: C. Cacciari and G. Clementini, p. 64.

Ostlie, D. A.: 1990, in The Numerical Modelling of Nonlinear Stellar Pulsations; Problems and Prospects, ed. J. R. Buchler (Kluwer, Dordrecht), p. 89.

Petersen, J. O.: 1973, Astronomy and Astrophysics 27, 89.

Rogers, F. J. and Iglesias, C. A.: 1992, Astrophysical Journal, Supplement Series 79 (in press, April).

Simon, N. R.: 1979, Astronomy and Astrophysics 75, 140.

Simon, N. R.: 1982, Astrophysical Journal, Letters to the Editor 260, L87.

Simon, N. R. Cox, A. N. and Hodson, S. W.: 1980, Astrophysical Journal 237, 550.

Simon, N. R. and Schmidt, E. G.: 1976, Astrophysical Journal 205, 162.

Stellingwerf, R. F.: 1975, Astrophysical Journal 195, 441.

Stellingwerf, R. F.: 1984, Astrophysical Journal 284, 712.

Stobie, R. S.: 1969, Monthly Notices of the RAS 144, 511.

Szabados, L.: 1988, in Multimode Stellar Pulsations, eds. G. Kovács, I. Szabados and B. Szeidl, Konkoly Observatory (Kultura: Budapest), p. 1.

Szeidl, B.: 1988, in Multimode Stellar Pulsations, eds. G. Kovács, L. Szabados and B. Szeidl, Konkoly Observatory (Kultura: Budapest), p. 45.

Walraven, Th., Walraven, J. and Balona, L. A.: 1992, Monthly Notices of the RAS 254, 59.

Wheeler, J. C., Sneden, C., and Truran, J. W.: 1989, Annual Review of Astronomy and Astrophysics 27, 279.

Zalewski, J.: 1992, these proceedings. 